\title{
Schema Mappings for the Web
}

\author{
François Scharffe \\ Digital Enterprise Research Institute \\ University of Innsbruck \\ francois.scharffe@deri.org
}

\begin{abstract}
Current solutions to data integration present many inconvenients. The bottleneck seems to be the impossible automation of the whole process. Human intervention will always be needed at some point, and the problem is to find where and how this intervention can be performed the most efficiently. In traditional mediator approaches the global schema and mappings between the global and local schemas are designed by hand. This is not the way to go if we want to see emerging a "semantic web". The collaborative development of one-to-one mappings driven by application needs has much more chance to rapidly create a network of schemas. We propose to build on top on this view, shifting the human intervention from the global schema elaboration to the one-to-one mapping between local schemas. This repartition of efforts associated with publication of the local mappings is the only solution if we want to see the deep web rising up and the semantic web vision becoming true. I propose to contribute to this paradigm at two levels. First, mappings between heterogeneous schemas must be universally understandable, as schema descriptions may be of various natures (XML, relational, Ontologies, Semi structured, ... ). An independant language able to model correspondences between two schemas is then needed. This language also serves as an exchange format for matching algorithms as well as graphical mapping tools. A global schema is still necessary in order to provide a unified view over resources. We propose in the following to study how from a network of related schemas can we extract a global schema together with the associated mapping rules.
\end{abstract}

